\title{
Genetic and phenotypical correlations, path analysis and genetic gain in two populations of corn with resistance to leaf spot, rust, and blight disease
}

\author{
A.S. Oliveira ${ }^{1}$, E.F. dos Reis ${ }^{2}$, A.P.O. Nogueira' ${ }^{1}$ D.B.O. Cardoso ${ }^{1}$ and \\ F.C. Juliatti ${ }^{1}$ \\ ${ }^{1}$ Universidade Federal de Uberlândia, Uberlândia, MG, Brasil \\ 2 Universidade Federal de Jataí, Jataí, GO, Brasil \\ Corresponding author: F.C. Juliatti \\ E-mail: juliatti@ufu.br
}

Genet. Mol. Res. 19 (2): gmr18408

Received June 16, 2019

Accepted May 23, 2020

Published June 30, 2020

DOI http://dx.doi.org/10.4238/gmr18408

\begin{abstract}
Phenotypic and genotypic correlations of agronomic traits with resistance to phytopathogens can help identify indirect selection criteria for corn yield. The Brazilian corn cultivar NAP5 is resistant to leaf spot and blight disease, while NAP7 is resistant to rust disease. Trials were conducted in two states from February to July 2015. The following traits were evaluated: GY - grain yield, PH - plant height, EH - ear height, MF - days to male flowering, NP number of plants, NE - number of ears, BP - number of broken plants, LP - number of lodged plants, KW - kernel weight, HT resistance to the fungus Exserohilum turcicum, WS (white spot) resistance to Pantoea ananatis, CS - resistance to corn stunt, PP resistance to Puccinia polysora and $\mathrm{CZ}$ - resistance to Cercospora zeae-maydis. The NAP5 population showed high genetic correlations between GY and PH, NP, NE and CS in Jataí, GO. In Uberlândia, MG, NAP5 presented high correlations between GY and PH, NP, NE, WS and CS. For the NAP7 population, genetic correlations were high between GY and CS in Uberlândia and between GY and NE in Jataí. Genetic gains for GY by direct selection ranged from 29 to $31 \%$. The influence of the location was low. NE had the greatest
\end{abstract}


effect on grain yield. For the NAP5 population, indirect selection for yield through selection for NE gave satisfactory genetic gain. The variable $\mathrm{NE}$ affected $\mathrm{KW}$, and could be used for indirect selection, though direct selection provides greater gains. The heritability estimate for GY (0.77) in the NAP5 population was higher than for NE (0.61). With direct selection, it is possible to obtain gains of up to $31 \%$ in GY. High genetic correlations were also observed in the NAP5 population between NE, GY and CS.

Key words: Foliar diseases; Full-sib plants; Genetic parameters; Direct and indirect selection; Zea mays

\section{INTRODUCTION}

The cultivation of a second corn crop (known as safrinha in Brazil) has now become one of the pillars of the Brazilian corn industry, resulting in high yields and becoming a major focus of current research. In the agricultural year of 2018/2019, the second corn harvest accounted for $75 \%$ of total corn production (CONAB, 2020). Due to the great importance and strong growth of the off-season corn crop, it is essential that breeding programs develop specific hybrids for this crop and develop strategies to improve efficiency in the selection of promising genotypes.

One of the important aspects of corn breeding programs is the number of selection methods that favor the identification of superior genotypes to obtain new hybrids with various desirable characteristics, such as high productivity and resistance to phytopathogens. The efficiency of selecting traits with low heritability, and that are difficult to measure, can be increased using correlated traits. Thus, knowledge of the correlations between the traits allows alternative selection strategies to be used during the breeding process so that genetic gain is maximized and that the selection process becomes more efficient by using traits with high heritability that are easy to measure, and thereby gaining several favorable traits at the same time (Cruz et al., 2012).

Correlations of a high magnitude between agronomic traits in corn have been reported (Toebe and Cargnelutti Filho, 2013, Cabral et al., 2016). Other research has shown correlation of a high magnitude between disease resistance and grain yield (Brito et al., 2007; Dudienas et al., 2013). Although correlation coefficients are very useful in quantifying the magnitude and direction of the influence the variable has in determining complex traits, they do not give the exact relative importance of the direct and indirect effects of these variables (Cruz et al., 2012). Studies about the splitting of the correlation coefficient are done using path analysis, which was developed by Wright (1921) and detailed by Li (1975).

Path analysis consists of quantifying the direct and indirect effects of the explanatory variables on a basic variable, whose estimates are obtained by means of regression equations in which the variables are previously standardized (Cruz et al., 2012). The path coefficient is a method that analyzes a system of multiple variables, linearly related and includes all the basic factors (causes) and their resulting variables (effects) (Li, 1956). Silva (2016) analyzing the correlation and trail analysis of 
agronomic and morphological characters in maize populations found an indirect correlation between lodging and stem breakage with production.

Using what is known about the correlations between genetic traits, as well as the direct and indirect effects, it is possible to achieve substantial gains using direct and indirect selection methods in breeding programs of corn. The objectives of this study were to evaluate two maize populations for phenotypic and genotypic correlations of agronomic traits and the resistance to important phytopathogens in the genetic improvement of corn; to carry out path analysis, having as a main trait grain yield; and to identify indirect selection criteria that lead to higher yields.

\section{MATERIAL AND METHODS}

The experiments were conducted from February to July 2015 (second crop) in two locations. The first being the Southeast Region of Goiás in Jataí, in the experimental area of the Federal University of Goiás, latitude 17 $52^{\prime} 53^{\prime \prime} \mathrm{S}$ and longitude $51^{\circ} 42^{\prime} 52^{\prime \prime} \mathrm{W}$, at an altitude of $676 \mathrm{~m}$. The second location was in Uberlandia within the Triângulo Mineiro Region, in the experimental area of the Federal University of Uberlândia, Glória Farm, on latitude 18 $58^{\prime} 52^{\prime \prime}$ S and longitude $48^{\circ} 12^{\prime} 24^{\prime \prime}$ W and an altitude of approximately $912 \mathrm{~m}$.

\section{Genetic material}

The populations of NAP5 corn (resistant to Exerohilum turcicum) and NAP7 (resistant to Physopella zeae) originated from a Technical-Scientific Cooperation project called NAP-Milho - Núcleo de Apoio a Pesquisa Milho (Corn Support Research Center), and was created by the Genetics Department of ESALQ / USP (Escola Superior de Agricultura "Luiz de Queiroz"/ Universidade de São Paulo) with the objective of identifying reliable sources of resistance to the main foliar diseases affecting corn. For this purpose, several public institutions (CNPMS / EMBRAPA, IAC - Agronomic Institute of Campinas, IAPAR - Agronomic Institute of Paraná, ESALQUSP, CENARGEN / EMBRAPA) and private companies (Zêneca Seeds, Colorado Seeds, AGRCERES) were involved.

In the agricultural year 1994/1995, 1273 accessions from an Active Germplasm Bank (BAG, CENARGEN / EMBRAPA) were evaluated at 13 sites in the states of Goiás, Mato Grosso, São Paulo and Paraná. Visual evaluations were carried out verifying the plants resistance to five foliar diseases and exclusively for each phytopathogen, with some accessions with low yield and poor plant architecture being excluded. The number of accessions that entered the composition of each population were 41 NAP5 and 34 NAP7. In the formation of each population a sample of seeds of each selected accession was used.

The lots were seeded in isolation at the Anhembi Experimental Station, with random pollination. After harvesting, a random sample of 1,000 seeds was taken from each population to plant the recombination cycle. From the recombination batches, about 200 plants were selected from each batch, which formed the half-sib progenies, 
thus initiating the intra-populational recurrent selection program. These populations are exotic, originating from several countries (Table 1) and more detailed information on these populations was reported by Mendes et al. (2015).

Table 1. Identification of two corn populations (NAP - Nucleus of Maize Research - University of São Paulo) obtained by recombination of accessions from the corn germplasm bank selected for specific resistance to leaf diseases.

\begin{tabular}{lllll}
\hline \multicolumn{7}{l}{ Population NAP-05 (resistance to Exserohilum turcicum) } & & \\
\hline BA 020 & BA 032 & CMS 49 & Honduras Gr. 13 & CMS 33 \\
BA 166 & RN 003 & WP 33 & Matahambre x Guapira & El Salvador Gr. 1 \\
CMS 457 & SE 030 & MG 060 & Templ. X 134M. Cristalino & CMS 44 \\
BA 018 & CNPH 1 & CMS 27 III & 134M. Bajio x Indon 027171 & CMS 47 \\
CMS 40 & BA 046 & BR 427 III & Bolívia I & Comp Cruz Cuzco \\
CMS 35 & BA 048 & Pipoca Timóteo & Zapalote Chico & BA 061 \\
SE 004 & BA 035 & Pipoca Miúdo & CMS 465 x Pool 33 QMP & --- \\
\hline Population NAP-07 (resistance to Physopella zeae) & & BA 035 \\
\hline AL 001 & CMS 44 & MEB I & COMITECO & CMS 38 \\
BA 188 & CMS 466 & CMS 30 & San Luiz Potosi 30 & SC 001 \\
CMS 457 & CMS 463 & BR 105 MI & Re. Dominicana 287 & Matahambre x Guapira \\
SC 005 & BA 036 & BA 019 & C. Belt x Rep. Dominicana & F36491-F2834T \\
CMS 49 & SE 028 & URG VA & CMS 465 Pool 33 Qpm & Comp. \\
\hline
\end{tabular}

To obtain the full-sib progenies from each population, two fields of half-sib progenies were planted, each consisting of 500 seeds. From these plant-to-plant crosses, 75 NAP5 and 100 NAP7 full-sib progenies were obtained to perform the experiments in Jataí, Goiás and Uberlândia, Minas Gerais.

\section{Experimental evaluation}

A randomized block design with three replications was used. Each experimental plot consisted of a row of plants $4 \mathrm{M}$ in length, spaced $0.9 \mathrm{M}$ apart. After thinning, the plots were left with 20 plants. In each plot, 5 plants were sampled and the severity of the five pathogens was determined. The severity assessments were performed from the onset of the first symptoms, with three evaluations and with a 14-day interval between them.

The evaluated traits were: $\mathrm{PH}$ - plant height $(\mathrm{cm}), \mathrm{EH}$ - ear height $(\mathrm{cm}), \mathrm{MF}$ male flowering (days), NE - number of ears, BP - number of broken plants, LP number of lodged plants, GY - grain yield $\left(\mathrm{t} . h a^{-1}\right)$, HT - Resistance to Exserohilum turcicum (AUDPC), WS (white spot) - resistance to Pantoea ananatis (AUDPC), CS resistance to corn stunt (AUDPC), PP - resistance to Puccinia polysora (AUDPC) and $\mathrm{CZ}$ - resistance to Cercospora zeae-maydis (AUDPC).

The severity assessments for WS and HT were made using the scales that were developed for the respective diseases and are presented in Annex 1D and 2D (Malagi et al., 2011; Lazaroto et al., 2012). For the CZ, PP and CS diseases, severity assessments were performed using the scale developed by Agroceres (1996) (Annex 3D), used to evaluate most leaf diseases in corn. 
The trait, grain yield (productivity) was corrected to the ideal number of plants, being 20, using the covariance correction methodology, suggested by Miranda Filho (Vencovsky and Barriga, 1992). From the analysis of variance and covariance for number of plants and yield, the linear regression coefficient (b) was obtained by $b=$ SPxy / SQx, where $x$ is number of plants and y is yield; SPxy the residual sum of products of the covariance analysis (plant number $\times$ grain weight); $S Q x$ is the residual sum of squares of the variance analysis for the number of plants.

Correction was performed at the level of total of plots, instead of the average number of treatments. Thus, the corrected grain weight of the field $(\mathrm{Pc})$ was obtained as follows:

$\mathrm{Pc}=\mathrm{P}-\mathrm{b}(\mathrm{x}-20)$, where: $\mathrm{Pc}=$ corrected grain weight; $\mathrm{P}=$ observed grain weight; $b=$ coefficient of linear regression of yield, in relation to variations in the number of plants; $x=$ number of observed plants.

The agronomic management of the experiments in the two trials was done according to recommendations provided for corn crops (Fancelli and Dourado-Neto, 2000). The analysis for each disease was performed through the area under the disease progress curve (AUDPC) and was used to describe the epidemic. In this case, based on severity assessments, one can establish a quantified "versus" time disease curve. According to Shaner and Finey (1977), AUDPC is calculated by the formula:

$$
A U D P C=\Sigma[(\mathrm{Yi}+\mathrm{Yi}-1) / 2 \times(\mathrm{Ti}+1-\mathrm{Ti})]
$$

where:

$$
\begin{aligned}
& Y_{i}=\text { severity of the disease in the } i \text {-th observation; } \\
& T_{i+1}-T_{i}=\text { is the time interval between two consecutive evaluations. }
\end{aligned}
$$

\section{Correlation analysis, path analysis and genetic gain}

The correlation (Wright 1921), path analysis ( $\mathrm{Li}$ 1975) and genetic gain estimates were performed for the variables evaluated according to the methodology described by Vencovsky and Barriga (1992) and Cruz et al. (2012). The correlation and genetic gain formulas are presented in Annex 1B. The significance of the phenotypic correlations was estimated by the t-test with (n-2) degrees of freedom, where $n$ corresponds to the number of evaluated genotypes. The significance of the genotypic correlations was evaluated via bootstrap with 5,000 simulations (Ferreira et al., 2008).

The diagnosis of multicollinearity involving the 12 variables was performed using the number of conditions (NC) method. In the NC method, the degree of multicollinearity was verified in the correlation matrix $\mathrm{X}^{\prime} \mathrm{X}$, which represents the ratio between the highest and the lowest eigenvalue of the correlation matrix (Montgomery and Peck, 1982). When the NC, resulting from this division, was less than or equal to 100 , it was considered that there was weak multicollinearity between the explanatory variables.

Then, the correlations of the other variables and the variable GY (dependent variable) were revealed to be direct or indirect effects, by path analysis, with a chain, 
establishing the cause and effect relationships between the characteristics, as described by Cruz et al. (2012). The interpretations of the path analysis results were done according to Singh and Chaudhary (1979), as quoted by Vencovsky and Barriga (1992).

For the genetic gain estimations, direct selection was performed for the variables (NE, GY, ET, WS, CS, PP and CZ), whereas indirect selection was performed for all the traits. In the NAP7 population, direct selection for NE was not performed, as there was no cause and effect relationship with GY. The proportion of selection used was $20 \%$. Statistical analysis was carried out with the aid of the Computational Program in Genetics and Statistics - Genes Program (Cruz, 2016).

\section{RESULTS AND DISCUSSION}

Tables 2, 3, 4 and 5 show the phenotypic and genotypic correlations of the NAP5 and NAP7 populations evaluated in Jataí and Uberlândia. In the interpretations of the correlations, three aspects that must be considered are magnitude, direction, and significance. Estimates of positive correlation coefficients indicate the tendency of one variable to increase when the other increases, negative correlations indicate that one variable tends to increase while the other decreases.

Table 2. The phenotypic (above diagonal) and genotypic (below diagonal) correlation coefficients of thirteen traits evaluated in seventy-five full-sib progenies from the NAP-5 corn population in Jataí, GO in 2015.

\begin{tabular}{|c|c|c|c|c|c|c|c|c|c|c|c|c|c|}
\hline Traits & PH & EH & MF & NP & NE & BP & LP & GY & ET & WS & CS & PP & $\mathbf{C Z}$ \\
\hline $\mathrm{PH}$ & & $0.79^{* *}$ & $0.08^{\mathrm{NS}}$ & $0.37 * *$ & $0.46^{* *}$ & $-0.09^{\mathrm{NS}}$ & $0.27 *$ & $0.49^{* *}$ & $-0.26^{*}$ & $0.03^{\mathrm{NS}}$ & $-0.52^{*} *$ & $-0.04^{\mathrm{NS}}$ & $-0.03^{\mathrm{NS}}$ \\
\hline $\mathrm{EH}$ & $0.83^{++}$ & & $0.42 * *$ & $0.40 * *$ & $0.32 * *$ & $0.09^{\mathrm{NS}}$ & $0.33^{* *}$ & $0.32 * *$ & $-0.36^{* *}$ & $-0.09^{\mathrm{NS}}$ & $-0.46^{* *}$ & $-0.0^{\mathrm{NS}}$ & $0.04^{\mathrm{NS}}$ \\
\hline MF & $0.10^{++}$ & $0.53^{++}$ & & $-0.08^{\mathrm{NS}}$ & $-0.32 * *$ & $-0.06^{\mathrm{NS}}$ & $0.28^{*}$ & $-0.34 * *$ & $-0.21^{\mathrm{NS}}$ & $-0.14^{\mathrm{NS}}$ & $0.23^{* *}$ & $-0.17^{\mathrm{NS}}$ & $0.05^{\mathrm{NS}}$ \\
\hline NP & $0.63^{++}$ & $0.69^{++}$ & $-0.04^{++}$ & & $0.57 * *$ & $0.32 * *$ & $0.18^{\mathrm{NS}}$ & $0.52^{* *}$ & $-0.19^{\mathrm{NS}}$ & $-0.26^{*}$ & $-0.60 * *$ & $0.05^{\mathrm{NS}}$ & $-0.02^{\mathrm{NS}}$ \\
\hline NE & $0.65^{++}$ & $0.42^{++}$ & $-0.39^{++}$ & $0.88^{++}$ & & $0.02^{\mathrm{NS}}$ & $0.01^{\mathrm{NS}}$ & $0.87 * *$ & $-0.36^{* *}$ & $0.02^{\mathrm{NS}}$ & $-0.59 * *$ & $0.02^{\mathrm{NS}}$ & $-0.08^{\mathrm{NS}}$ \\
\hline BP & $-0.11^{++}$ & $0.16^{++}$ & $-0.06^{++}$ & $0.32^{++}$ & $0.09^{++}$ & & $-0.43^{* *}$ & $0.07^{\mathrm{NS}}$ & $0.03^{\mathrm{NS}}$ & $-0.14^{\mathrm{NS}}$ & $-0.04^{\mathrm{NS}}$ & $0.19^{\mathrm{NS}}$ & $0.08^{\mathrm{NS}}$ \\
\hline LP & $0.41^{++}$ & $0.53^{++}$ & $0.40^{++}$ & $0.18^{++}$ & $0.08^{++}$ & $-0.74^{++}$ & & $-0.05^{\mathrm{NS}}$ & $-0.09^{\mathrm{NS}}$ & $-0.08^{\mathrm{NS}}$ & $-0.14^{\mathrm{NS}}$ & $-0.09^{\mathrm{NS}}$ & $0.06^{\mathrm{NS}}$ \\
\hline GY & $0.62^{++}$ & $0.38^{++}$ & $-0.41^{++}$ & $0.52^{++}$ & $1.04^{++}$ & $0.19^{++}$ & $0.06^{++}$ & & $-0.32 * *$ & $-0.12^{\mathrm{NS}}$ & $-0.60 * *$ & $-0.01^{\mathrm{NS}}$ & $-0.13^{\mathrm{NS}}$ \\
\hline ET & $-0.30^{++}$ & $-0.39^{++}$ & $-0.27^{++}$ & $-0.19^{++}$ & $-0.47^{++}$ & $0.04^{++}$ & $-0.19^{++}$ & $-0.39^{++}$ & & $-0.01^{\mathrm{NS}}$ & $0.29 *$ & $0.32^{* *} *$ & $-0.04^{\mathrm{NS}}$ \\
\hline WS & $0.04^{++}$ & $-0.09^{++}$ & $-0.17^{++}$ & $-0.26^{++}$ & $0.05^{++}$ & $-0.28^{++}$ & $-0.11^{++}$ & $-0.13^{++}$ & $-0.03^{++}$ & & $-0.01^{\mathrm{NS}}$ & $0.34 * *$ & $0.35^{* * *}$ \\
\hline CS & $-0.73^{++}$ & $-0.59^{++}$ & $0.29^{++}$ & $-0.60^{++}$ & $-0.82^{++}$ & $-0.16^{++}$ & $-0.29^{++}$ & $-0.74^{++}$ & $0.30^{++}$ & $-0.02^{++}$ & & $-0.08^{\mathrm{NS}}$ & $0.18^{\mathrm{NS}}$ \\
\hline $\mathrm{PP}$ & $-0.10^{++}$ & $-0.11^{++}$ & $-0.24^{++}$ & $0.05^{++}$ & $-0.00^{++}$ & $0.22^{++}$ & $-0.20^{++}$ & $-0.03^{++}$ & $0.46^{++}$ & $0.52^{++}$ & $-0.12^{++}$ & & $0.07^{\mathrm{NS}}$ \\
\hline $\mathrm{CZ}$ & $0.01^{++}$ & $0.09^{++}$ & $0.07^{++}$ & $-0.02^{++}$ & $-0.12^{++}$ & $0.15^{++}$ & $0.00^{++}$ & $-0.19^{++}$ & $-0.04^{++}$ & $0.52^{++}$ & $0.24^{++}$ & $0.08^{++}$ & \\
\hline
\end{tabular}

The correlation that can be directly measured from the measures of two traits is phenotypic, derived from genetic and environmental causes, but only the genetic ones involve an association of inheritable nature (Cruz et al., 2012). In the two populations of both evaluated sites, it was observed that, predominantly, the genotypic correlations were higher than the phenotypic correlations and of the same signal, indicating a lower environmental influence on the expression of the traits. These results are consistent with those obtained in other corn studies (Cabral et al., 2016).

The genetic correlations observed in the NAP5 population between the PH and CS traits were of a high magnitude, significant and of opposite direction, that is, the 
greater the severity of corn stunt, the lower the plant height. The reduction in plant height occurs because plant diseases are systemic diseases that affect the development of the plants, reducing the size of the internodes and consequently lowering the plants' PH and EH (Oliveira et al., 2004). In the NAP7 population, the correlation between PH and CS was of a high magnitude in the experiment evaluated in Uberlândia - MG and of low magnitude in Jataí - GO.

Table 3. The phenotypic (above diagonal) and genotypic (below diagonal) correlation coefficients of thirteen traits evaluated in seventy-five full-sib progenies of the exotic NAP-5 corn population in Uberlândia, MG in 2015.

\begin{tabular}{|c|c|c|c|c|c|c|c|c|c|c|c|c|c|}
\hline Traits & PH & EH & MF & NP & $\mathbf{N E}$ & BP & LP & GY & ET & WS & CS & PP & $\mathbf{C Z}$ \\
\hline$\overline{\mathrm{PH}}$ & & $0.79 * *$ & $0.11^{\mathrm{NS}}$ & $0.46^{* *}$ & $0.46^{* *}$ & $0.01^{\mathrm{NS}}$ & $0.38^{* *}$ & 0.47 ** & $-0.26^{*}$ & $0.03^{\mathrm{NS}}$ & $-0.55^{* * *}$ & $0.05^{\mathrm{NS}}$ & $-0.05^{\mathrm{NS}}$ \\
\hline EH & $0.82^{++}$ & & $0.47 * *$ & $0.24^{*}$ & $0.23^{*}$ & $0.22^{\mathrm{NS}}$ & $0.47 * *$ & $0.21^{\mathrm{NS}}$ & $-0.25^{*}$ & $-0.02^{\mathrm{NS}}$ & $-0.27^{*}$ & $0.00^{\mathrm{NS}}$ & $0.04^{\mathrm{NS}}$ \\
\hline MF & $0.15^{++}$ & $0.54^{++}$ & & $-0.27 *$ & $-0.33^{* *}$ & $0.03^{\mathrm{NS}}$ & $0.43^{* *}$ & $-0.26^{*}$ & $-0.12^{\mathrm{NS}}$ & $-0.12^{\mathrm{NS}}$ & $0.28 *$ & $-0.26^{*}$ & $0.01^{\mathrm{NS}}$ \\
\hline NP & $0.82^{++}$ & $0.37^{++}$ & $-0.49^{++}$ & & - & $0.04^{\mathrm{NS}}$ & $0.08^{\mathrm{NS}}$ & $0.36^{\mathrm{NS}}$ & $-0.08^{\mathrm{NS}}$ & $0.24^{\mathrm{NS}}$ & $-0.54^{\mathrm{NS}}$ & $0.20^{\mathrm{NS}}$ & $-0.18^{\mathrm{NS}}$ \\
\hline $\mathrm{NE}$ & $0.65^{++}$ & $0.30^{++}$ & $-0.46^{++}$ & - & & $-0.05^{\mathrm{NS}}$ & $0.02^{\mathrm{NS}}$ & $0.56^{* * *}$ & $-0.27 *$ & $0.10^{\mathrm{NS}}$ & $-0.57 * *$ & $0.16^{\mathrm{NS}}$ & $-0.08^{\mathrm{NS}}$ \\
\hline BP & $0.15^{++}$ & $0.44^{++}$ & $-0.02^{++}$ & $0.09^{++}$ & $-0.15^{++}$ & & $-0.11^{\mathrm{NS}}$ & $-0.04^{\mathrm{NS}}$ & $-0.02^{\mathrm{NS}}$ & $0.22^{\mathrm{NS}}$ & $0.01^{\mathrm{NS}}$ & $0.07^{\mathrm{NS}}$ & $-0.01^{\mathrm{NS}}$ \\
\hline LP & $0.81^{++}$ & $0.89^{++}$ & $0.71^{++}$ & $-0.11^{++}$ & $0.19^{++}$ & $0.06^{++}$ & & $0.16^{\mathrm{NS}}$ & -0.31 ** & $-0.04^{\mathrm{NS}}$ & $-0.17^{\mathrm{NS}}$ & $-0.24^{*}$ & $-0.07^{\mathrm{NS}}$ \\
\hline GY & $0.60^{++}$ & $0.21^{++}$ & $-0.30^{++}$ & $0.51^{++}$ & $0.74^{++}$ & $-0.02^{++}$ & $0.46^{++}$ & & $-0.36^{* *}$ & $0.05^{\mathrm{NS}}$ & $-0.62^{* *}$ & $0.05^{\mathrm{NS}}$ & $-0.11^{\mathrm{NS}}$ \\
\hline ET & $-0.31^{++}$ & $-0.28^{++}$ & $-0.14^{++}$ & $-0.09^{++}$ & $-0.37^{++}$ & $-0.09^{++}$ & $-0.49^{++}$ & $-0.44^{++}$ & & $0.09^{\mathrm{NS}}$ & $0.29 *$ & $-0.07^{\mathrm{NS}}$ & $-0.11^{\mathrm{NS}}$ \\
\hline WS & $0.07^{++}$ & $-0.01^{++}$ & $-0.13^{++}$ & $0.59^{++}$ & $0.18^{++}$ & $0.40^{++}$ & $-0.04^{++}$ & $0.05^{++}$ & $0.10^{++}$ & & $-0.17^{\mathrm{NS}}$ & $0.14^{\mathrm{NS}}$ & $-0.09^{\mathrm{NS}}$ \\
\hline CS & $-0.69^{++}$ & $-0.31^{++}$ & $0.34^{++}$ & $-0.99^{++}$ & $-1.01^{++}$ & $-0.03^{++}$ & $-0.36^{++}$ & $-0.82^{++}$ & $0.38^{++}$ & $-0.21^{++}$ & & $-0.31^{* * *}$ & $0.08^{\mathrm{NS}}$ \\
\hline PP & $-0.09^{++}$ & $-0.12^{++}$ & $-0.44^{++}$ & $0.32^{++}$ & $0.12^{++}$ & $0.23^{++}$ & $-0.58^{++}$ & $0.01^{++}$ & $-0.07^{++}$ & $0.36^{++}$ & $-0.63^{++}$ & & $0.00^{\mathrm{NS}}$ \\
\hline $\mathrm{CZ}$ & $-0.10^{++}$ & $0.06^{++}$ & $0.02^{++}$ & $-0.45^{++}$ & $-0.24^{++}$ & $-0.16^{++}$ & $-0.35^{++}$ & $-0.29^{++}$ & $-0.08^{++}$ & $-0.09^{++}$ & $0.15^{++}$ & $-0.20^{++}$ & \\
\hline
\end{tabular}

*: significant at the $1 \%$ probability level by the t-test. ${ }^{N s}$ : not significant. ${ }^{++}$: significant at $1 \%$ by the bootstrap method with 5 thousand simulations. PH: plant height; EH: ear height; MF: male flowering; NP: number of plants; NE: number of ears; BP: number of broken plants; LP: number of lodged plants; GY: grain yield; ET: Resistance to Exserohilum turcicum, WS: resistance to Pantoea ananatis (white spot), CS: resistance to corn stunt, PP: resistance to Puccinia polysora and CZ: resistance to Cercospora zeae-maydis. *: significant at the $\%$ probability level by the t-test.

Table 4. The phenotypic (above diagonal) and genotypic (below diagonal) correlation coefficients of thirteen traits evaluated in seventy-five full-sib progenies of the NAP-7 corn population in Jataí, GO in 2015.

\begin{tabular}{|c|c|c|c|c|c|c|c|c|c|c|c|c|c|}
\hline Traits & PH & EH & MF & NP & NE & BP & LP & GY & ET & WS & CS & PP & $\mathbf{C Z}$ \\
\hline $\mathrm{PH}$ & & $0.80 * *$ & $0.23 *$ & $0.24 *$ & 0.25 & $0.17^{\mathrm{NS}}$ & $0.14^{\mathrm{NS}}$ & $0.08^{\mathrm{NS}}$ & $-0.13^{\mathrm{NS}}$ & $0.19^{\mathrm{NS}}$ & $-0.13^{\mathrm{NS}}$ & $-0.05^{\mathrm{NS}}$ & $-0.20^{*}$ \\
\hline EH & $0.84^{++}$ & & $0.33 * *$ & $0.18^{\mathrm{NS}}$ & 0.23 & $0.24 *$ & $0.16^{\mathrm{NS}}$ & $0.13^{\mathrm{NS}}$ & $-0.18^{\mathrm{NS}}$ & $0.15^{\mathrm{NS}}$ & $-0.19^{\mathrm{NS}}$ & $-0.02^{\mathrm{NS}}$ & $-0.20 *$ \\
\hline MF & $0.25^{++}$ & $0.38^{++}$ & & $-0.15^{\mathrm{NS}}$ & -0.06 & $0.05^{\mathrm{NS}}$ & $0.07^{\mathrm{NS}}$ & $0.06^{\mathrm{NS}}$ & $-0.19^{\mathrm{NS}}$ & $-0.08^{\mathrm{NS}}$ & $0.00^{\mathrm{NS}}$ & $-0.12^{\mathrm{NS}}$ & $0.01^{\mathrm{NS}}$ \\
\hline NP & $0.30^{++}$ & $0.23^{++}$ & $-0.18^{++}$ & & $0.51 * *$ & $0.28 * *$ & $0.29^{*}$ & $0.21^{*}$ & $-0.04^{\mathrm{NS}}$ & $0.05^{\mathrm{NS}}$ & $-0.27 * *$ & $0.21^{*}$ & $0.12^{\mathrm{NS}}$ \\
\hline $\mathrm{NE}$ & $0.39^{++}$ & $0.35^{++}$ & $-0.10^{++}$ & $0.77^{++}$ & & $0.10^{\mathrm{NS}}$ & $0.06^{\mathrm{NS}}$ & $0.59 * *$ & $-0.17^{\mathrm{NS}}$ & $0.14^{\mathrm{NS}}$ & $-0.27 * *$ & $0.14^{\mathrm{NS}}$ & $0.04^{\mathrm{NS}}$ \\
\hline BP & $0.20^{++}$ & $0.33^{++}$ & $0.14^{++}$ & $0.19^{++}$ & $0.08^{++}$ & & $-0.49^{* *}$ & $0.09^{\mathrm{NS}}$ & $-0.03^{\mathrm{NS}}$ & $-0.11^{\mathrm{NS}}$ & $-0.12^{\mathrm{NS}}$ & $0.14^{\mathrm{NS}}$ & $0.17^{\mathrm{NS}}$ \\
\hline LP & $0.21^{++}$ & $0.25^{++}$ & $0.20^{++}$ & $0.02^{++}$ & $0.18^{++}$ & $-0.87^{++}$ & & $-0.06^{\mathrm{NS}}$ & $-0.08^{\mathrm{NS}}$ & $0.16^{\mathrm{NS}}$ & $-0.09^{\mathrm{NS}}$ & $-0.06^{\mathrm{NS}}$ & $-0.07^{\mathrm{NS}}$ \\
\hline GY & $0.10^{++}$ & $0.16^{++}$ & $0.08^{++}$ & $0.34^{++}$ & $0.89^{++}$ & $0.13^{++}$ & $-0.05^{++}$ & & $-0.19^{\mathrm{NS}}$ & $-0.10^{\mathrm{NS}}$ & $-0.07^{\mathrm{NS}}$ & $0.02^{\mathrm{NS}}$ & $-0.07^{\mathrm{NS}}$ \\
\hline ET & $-0.16^{++}$ & $-0.22^{++}$ & $-0.20^{++}$ & $-0.10^{++}$ & $-0.22^{++}$ & $-0.07^{++}$ & $-0.18^{++}$ & $-0.21^{++}$ & & $0.23 *$ & $0.09^{\mathrm{NS}}$ & $-0.09^{\mathrm{NS}}$ & $0.11^{\mathrm{NS}}$ \\
\hline WS & $0.22^{++}$ & $0.17^{++}$ & $-0.14^{++}$ & $0.11^{++}$ & $0.20^{++}$ & $-0.16^{++}$ & $0.31^{++}$ & $-0.11^{++}$ & $0.26^{++}$ & & $0.24 *$ & $0.08^{\mathrm{NS}}$ & $-0.20 *$ \\
\hline CS & $-0.19^{++}$ & $-0.25^{++}$ & $-0.01^{++}$ & $-0.47^{++}$ & $-0.44^{++}$ & $-0.23^{++}$ & $-0.15^{++}$ & $-0.08^{++}$ & $0.12^{++}$ & $0.33^{++}$ & & $-0.26^{* *}$ & $-0.10^{\mathrm{NS}}$ \\
\hline PP & $-0.13^{++}$ & $-0.07^{++}$ & $-0.19^{++}$ & $0.42^{++}$ & $0.18^{++}$ & $0.22^{++}$ & $-0.01^{++}$ & $-0.02^{++}$ & $-0.19^{++}$ & $0.13^{++}$ & $-0.44^{++}$ & & $0.13^{\mathrm{NS}}$ \\
\hline $\mathrm{CZ}$ & $-0.29^{++}$ & $-0.29^{++}$ & $0.00^{++}$ & $0.30^{++}$ & $0.03^{++}$ & $0.29^{++}$ & $-0.09^{++}$ & $-0.07^{++}$ & $0.11^{++}$ & $-0.34^{++}$ & $-0.13^{++}$ & $0.11^{++}$ & \\
\hline
\end{tabular}

${ }^{* *}$ : significant at the $1 \%$ probability level by the t-test. ${ }^{N S}$ : not significant. ${ }^{++}$: significant at $1 \%$ by the bootstrap method with 5 thousand simulations. PH: plant height; EH: ear height; MF: male flowering; NP: number of plants; NE: number of ears; BP: number of broken plants; LP: number of lodged plants; GY: Grain yield ; ET: Resistance to Exserohilum turcicum, WS: resistance to Pantoea ananatis (white spot), CS: resistance to corn stunt, PP: resistance to Puccinia polysora and CZ: resistance to Cercospora zeae-maydis. *: significant at the $\%$ probability level by the t-test. 
Table 5. The phenotypic (above diagonal) and genotypic (below diagonal) correlation coefficients of thirteen traits evaluated in seventy-five full-sib progenies of the NAP-7 corn population in Uberlândia, MG in 2015.

\begin{tabular}{|c|c|c|c|c|c|c|c|c|c|c|c|c|c|}
\hline Traits & PH & EH & MF & NP & NE & BP & LP & GY & ET & WS & CS & PP & $\mathbf{C Z}$ \\
\hline $\mathrm{PH}$ & & $0.84^{* *}$ & $0.22 *$ & $0.19^{\mathrm{NS}}$ & $0.46^{* *}$ & $0.32 * *$ & $0.05^{\mathrm{NS}}$ & $0.29 * *$ & $0.05^{\mathrm{NS}}$ & $0.14^{\mathrm{NS}}$ & $-0.50^{* *}$ & $-0.13^{\mathrm{NS}}$ & $-0.01^{\mathrm{NS}}$ \\
\hline $\mathrm{EH}$ & $0.87^{++}$ & & $0.44 * *$ & $0.04^{\mathrm{NS}}$ & $0.36 * *$ & $0.51^{* *}$ & $0.11^{\mathrm{NS}}$ & $0.21 *$ & $-0.02^{\mathrm{NS}}$ & $0.18^{\mathrm{NS}}$ & $-0.48 * *$ & $-0.04^{\mathrm{NS}}$ & $-0.03^{\mathrm{NS}}$ \\
\hline MF & $0.37^{++}$ & $0.60^{++}$ & & $-0.18^{\mathrm{NS}}$ & $0.02^{\mathrm{NS}}$ & $0.30 * *$ & $0.28 * *$ & $-0.03^{\mathrm{NS}}$ & $-0.12^{\mathrm{NS}}$ & $0.03^{\mathrm{NS}}$ & $-0.21 *$ & $-0.08^{\mathrm{NS}}$ & $-0.03^{\mathrm{NS}}$ \\
\hline NP & $0.25^{++}$ & $0.05^{++}$ & $-0.31^{++}$ & & $0.44^{* *}$ & $-0.13^{\mathrm{NS}}$ & $-0.07^{\mathrm{NS}}$ & $0.12^{\mathrm{NS}}$ & $-0.13^{\mathrm{NS}}$ & $-0.03^{\mathrm{NS}}$ & $-0.26 * *$ & $-0.24 * *$ & $-0.14^{\mathrm{NS}}$ \\
\hline $\mathrm{NE}$ & $0.63^{++}$ & $0.47^{++}$ & $0.13^{++}$ & $0.63^{++}$ & & $0.04^{\mathrm{NS}}$ & $0.04^{\mathrm{NS}}$ & $0.22 *$ & $-0.06^{\mathrm{NS}}$ & $0.02^{\mathrm{NS}}$ & $-0.37 * *$ & $-0.07^{\mathrm{NS}}$ & $-0.09^{\mathrm{NS}}$ \\
\hline $\mathrm{BP}$ & $0.63^{++}$ & $1.00^{++}$ & $0.78^{++}$ & $-0.75^{++}$ & $0.03^{++}$ & & $-0.01^{\mathrm{NS}}$ & $-0.12^{\mathrm{NS}}$ & $0.01^{\mathrm{NS}}$ & $0.12^{\mathrm{NS}}$ & $-0.24 *$ & $-0.11^{\mathrm{NS}}$ & $0.03^{\mathrm{NS}}$ \\
\hline LP & $0.27^{++}$ & $0.28^{++}$ & $0.48^{++}$ & $-0.53^{++}$ & $0.30^{++}$ & $0.67^{++}$ & & $0.21 *$ & $-0.06^{\mathrm{NS}}$ & $0.00^{\mathrm{NS}}$ & $-0.11^{\mathrm{NS}}$ & $-0.30^{* *}$ & $-0.03^{\mathrm{NS}}$ \\
\hline GY & $0.34^{++}$ & $0.24^{++}$ & $-0.04^{++}$ & $0.27^{++}$ & $0.30^{++}$ & $-0.25^{++}$ & $0.46^{++}$ & & $-0.20 *$ & $-0.19^{\mathrm{NS}}$ & $-0.30 * *$ & $-0.02^{\mathrm{NS}}$ & $-0.01^{\mathrm{NS}}$ \\
\hline ET & $0.02^{++}$ & $-0.07^{++}$ & $-0.15^{++}$ & $-0.26^{++}$ & $-0.07^{++}$ & $-0.16^{++}$ & $-0.14^{++}$ & $-0.26^{++}$ & & $0.09^{\mathrm{NS}}$ & $0.07^{\mathrm{NS}}$ & $-0.06^{\mathrm{NS}}$ & $-0.05^{\mathrm{NS}}$ \\
\hline WS & $0.23^{++}$ & $0.25^{++}$ & $0.04^{++}$ & $0.01^{++}$ & $0.01^{++}$ & $0.13^{++}$ & $-0.12^{++}$ & $-0.21^{++}$ & $0.14^{++}$ & & $-0.07^{\mathrm{NS}}$ & $-0.05^{\mathrm{NS}}$ & $-0.19^{\mathrm{NS}}$ \\
\hline $\mathrm{CS}$ & $-0.64^{++}$ & $-0.63^{++}$ & $-0.34^{++}$ & $-0.54^{++}$ & $-0.52^{++}$ & $-0.57^{++}$ & $-0.28^{++}$ & $-0.40^{++}$ & $0.07^{++}$ & $-0.18^{++}$ & & $0.10^{\mathrm{NS}}$ & $-0.11^{\mathrm{NS}}$ \\
\hline PP & $-0.14^{++}$ & $0.07^{++}$ & $-0.04^{++}$ & $-0.77^{++}$ & $-0.14^{++}$ & $-0.49^{++}$ & $-0.44^{++}$ & $-0.01^{++}$ & $-0.04^{++}$ & $0.01^{++}$ & $0.07^{++}$ & & $0.09^{\mathrm{NS}}$ \\
\hline $\mathrm{CZ}$ & $0.01^{++}$ & $-0.04^{++}$ & $-0.01^{++}$ & $-0.51^{++}$ & $-0.23^{++}$ & $-0.07^{++}$ & $-0.09^{++}$ & $-0.03^{++}$ & $-0.12^{++}$ & $-0.34^{++}$ & $-0.28^{++}$ & $0.10^{++}$ & \\
\hline
\end{tabular}

Table 6. Direct and indirect effects of the traits plant height (PH), height of ear (EH), male flowering (MF), number of plants (NP), number of ears (NE), number of broken plants (BP), number of lodged plants (LP), resistance to Exserohilum turcicum (ET), resistance to Pantoea ananatis (WS-white spot), resistance to corn stunt (CS), resistance to Puccinia polysora (PP) and resistance to Cercospora zeae (CZ), on grain yield (GY), coefficient of determination of the path analysis and effect of the residual variable of the NAP5 population in Jataí, GO in 2015.

\begin{tabular}{|c|c|c|c|c|c|c|c|c|c|c|c|c|}
\hline Effects & PH & EH & MF & NP & NE & BP & LP & ET & WS & CS & PP & $\mathbf{C Z}$ \\
\hline Direct Effect GY & 0.230 & -0.120 & -0.061 & -0.086 & 0.737 & 0.033 & -0.081 & -0.047 & -0.210 & -0.144 & 0.023 & 0.044 \\
\hline Indirect effect PH & & 0.183 & 0.018 & 0.085 & 0.106 & -0.020 & 0.062 & -0.059 & 0.006 & -0.120 & -0.008 & -0.007 \\
\hline Indirect effect EH & -0.095 & & -0.050 & -0.048 & -0.038 & -0.011 & -0.040 & 0.043 & 0.011 & 0.055 & 0.007 & -0.005 \\
\hline Indirect effect MF & -0.005 & -0.025 & & 0.005 & 0.019 & 0.004 & -0.017 & 0.013 & 0.009 & -0.014 & 0.011 & -0.003 \\
\hline Indirect effect NP & -0.032 & -0.034 & 0.007 & & -0.049 & -0.028 & -0.015 & 0.016 & 0.023 & 0.051 & -0.005 & 0.002 \\
\hline Indirect effect NE & 0.339 & 0.237 & -0.233 & 0.420 & & 0.015 & 0.010 & -0.266 & 0.015 & -0.435 & 0.017 & -0.060 \\
\hline Indirect effect BP & -0.003 & 0.003 & -0.002 & 0.011 & 0.001 & & -0.014 & 0.001 & -0.005 & -0.001 & 0.006 & 0.003 \\
\hline Indirect effect LP & -0.022 & -0.027 & -0.022 & -0.014 & -0.001 & 0.034 & & 0.007 & 0.007 & 0.012 & 0.007 & -0.005 \\
\hline Indirect effect HT & 0.012 & 0.017 & 0.010 & 0.009 & 0.017 & -0.001 & 0.004 & & 0.001 & -0.014 & -0.015 & 0.002 \\
\hline Indirect effect WS & -0.006 & 0.019 & 0.030 & 0.056 & -0.004 & 0.030 & 0.017 & 0.003 & & 0.002 & -0.071 & -0.073 \\
\hline Indirect effect CS & 0.075 & 0.066 & -0.033 & 0.086 & 0.085 & 0.006 & 0.021 & -0.042 & 0.001 & & 0.011 & -0.026 \\
\hline Indirect effect PP & -0.001 & -0.001 & -0.004 & 0.001 & 0.001 & 0.004 & -0.002 & 0.007 & 0.008 & -0.002 & & 0.001 \\
\hline Indirect effect $\mathrm{CZ}$ & -0.001 & 0.002 & 0.002 & -0.001 & -0.004 & 0.003 & 0.002 & -0.002 & 0.015 & 0.008 & 0.003 & \\
\hline Total & 0.493 & 0.319 & -0.338 & 0.524 & 0.869 & 0.071 & -0.052 & -0.325 & -0.120 & -0.603 & -0.013 & -0.128 \\
\hline Coefficient of determination & 0.820 & & & & & & & & & & & \\
\hline Effects of the residue variable & 0.424 & & & & & & & & & & & \\
\hline
\end{tabular}

High genetic correlations were also observed in the NAP5 population between the NE, GY and CS traits (Table 2,3,6 and 7). The correlations between NE and GY were positive, indicating that the higher the ear number the higher the grain yield. However, the NE and KW traits were affected by the severity of CS and negative correlations were observed between the disease and these two traits. As previously described, CS is a systemic disease and in addition to reducing the size of the plant it also reduces grain yield in the ears and consequently affects the final grain yield (Cota et al., 2015). 
However, although the populations show significant correlations and high magnitude between the all traits (Table 6 and 7), these results do not give us the exact relative importance of the direct and indirect effects (Cruz et al., 2012). For greater efficiency in the selection process, it is important to quantify the direct and indirect effects of the explanatory variables on the main variable. Thus, path analysis is used as it helps the geneticist to quantify these effects.

Table 7. Direct and indirect effects of traits of corn plant height $(\mathrm{PH})$, height of ears $(\mathrm{EH})$, days of male flowering (MF), number of plants (NP), number of ears (NE), number of broken plants (BP), number of lodged plants (LP) resistance to Exserohilum turcicum (ET), resistance to Pantoea ananatis (white spot) (WS) resistance to corn stunt (CS), resistance to Puccinia polysora (PP) and resistance to Cercospora zeae (CZ) on grain yield, coefficient of determination of the path analysis and effect of the residual variable of the NAP5 population in Uberlândia, MG in 2015.

\begin{tabular}{|c|c|c|c|c|c|c|c|c|c|c|c|c|}
\hline Effect & PH & EH & MF & $\mathbf{N P}$ & NE & BP & LP & HT & WS & $\mathrm{CS}$ & $\mathbf{P P}$ & $\mathbf{C Z}$ \\
\hline Direct effect GY & 0.295 & -0.151 & -0.144 & -0.154 & 0.273 & 0.036 & 0.039 & -0.181 & -0.013 & -0.359 & -0.130 & -0.081 \\
\hline Indirect Effect PH & & 0.234 & 0.032 & 0.136 & 0.137 & 0.002 & 0.113 & -0.078 & 0.010 & -0.163 & 0.016 & -0.015 \\
\hline Indirect Effect EH & -0.120 & & -0.071 & -0.036 & -0.035 & -0.033 & -0.071 & 0.038 & 0.003 & 0.040 & 0.001 & -0.005 \\
\hline Indirect Effect MF & -0.015 & -0.067 & & 0.039 & 0.047 & -0.005 & -0.062 & 0.018 & 0.017 & -0.041 & 0.037 & -0.001 \\
\hline Indirect Effect NP & -0.071 & -0.037 & 0.042 & & -0.099 & -0.007 & -0.013 & 0.013 & -0.036 & 0.084 & -0.031 & 0.027 \\
\hline Indirect Effect NE & 0.126 & 0.063 & -0.090 & 0.175 & & -0.013 & 0.005 & -0.073 & 0.027 & -0.156 & 0.043 & -0.023 \\
\hline Indirect Effect BP & 0.000 & 0.008 & 0.001 & 0.002 & -0.002 & & -0.004 & -0.001 & 0.008 & 0.000 & 0.003 & 0.000 \\
\hline Indirect Effect LP & 0.015 & 0.018 & 0.017 & 0.003 & 0.001 & -0.004 & & -0.012 & -0.001 & -0.007 & -0.009 & -0.003 \\
\hline Indirect Effect HT & 0.048 & 0.045 & 0.022 & 0.015 & 0.048 & 0.004 & 0.057 & & -0.016 & -0.052 & 0.013 & 0.021 \\
\hline Indirect Effect WS & 0.000 & 0.000 & 0.002 & -0.003 & -0.001 & -0.003 & 0.001 & -0.001 & & 0.002 & -0.002 & 0.001 \\
\hline Indirect Effect CS & 0.199 & 0.096 & -0.102 & 0.195 & 0.206 & -0.005 & 0.061 & -0.104 & 0.061 & & 0.112 & -0.030 \\
\hline Indirect Effect PP & -0.007 & 0.001 & 0.033 & -0.026 & -0.020 & -0.010 & 0.031 & 0.010 & -0.018 & 0.040 & & 0.001 \\
\hline Indirect Effect CZ & 0.004 & -0.003 & -0.001 & 0.014 & 0.007 & 0.001 & 0.006 & 0.009 & 0.008 & -0.007 & 0.000 & \\
\hline Total & 0.473 & 0.206 & -0.259 & 0.360 & 0.561 & -0.036 & 0.162 & -0.363 & 0.047 & -0.618 & 0.053 & -0.109 \\
\hline Coefficient of determ & 0.537 & & & & & & & & & & & \\
\hline Effect of the residue variable & 0.681 & & & & & & & & & & & \\
\hline
\end{tabular}

To obtain reliability in the results presented by the path analysis it is important that the evaluated traits do not present high collinearity. Based on the number of conditions proposed by Montgomery and Peck (1982), the correlation matrix X'X with the thirteen variables presented low multicollinearity with values from 32 to 50, not constituting a problem for path analysis.

Tables 6 and 7 show the direct and indirect effects of the variables on grain yield. The highest coefficient of determination was observed for the NAP5 population evaluated in the location Jataí, GO with a value equal to 0.82 , signifying that $82 \%$ of the variation of the dependent variable GY in the model is explained by the variables used in the causal diagram. For the NAP7 population, path analysis was not presented because the correlation coefficients between the explanatory variables and the main variable (GY) were of low magnitude.

Although the NP, NE and CS variables showed high correlations on GY in at least one of the evaluated sites, only NE had a cause and effect relationship with GY, because its direct effects evaluated in the NAP5 (Jataí-GO) population were higher than the effect of the residual variable. This result reveals how the study of correlations can cause the researcher to make mistakes during the selection process, and also demonstrates how important it is to reveal whether the correlations have direct or indirect effects. Thus, it can be inferred that both the direct selection of more productive 
progenies and the indirect selection of progenies that present a higher NE are efficient in increasing grain yield of corn for the NAP5 population.

Regarding the variables related to resistance to disease, CS showed a high correlation with GY in both evaluated locations. However, the direct effect was lower than the residual effect, and the indirect effect of NE in the Jataí test was higher than residual effect, again confirming the strong influence of this variable on GY. The estimates of genetic gain of the NAP5 and NAP7 populations evaluated in the locations Jataí, GO and Uberlândia, MG are presented in Tables 8 and 9, however the NAP7 population estimates were only made for direct selection gain due to the low magnitude of the correlations observed in this population.

Table 8. Estimates of genetic gain in percentage by direct selection method for NE, GY, ET, WS, CS, PP and $\mathrm{CZ}$ and indirect for 13 traits evaluated, average population (Xo) and average of the 15 families selected (Xs) in the population of NAP-5 corn in Jataí GO and Uberlândia, MG in 2015.

\begin{tabular}{|c|c|c|c|c|c|c|c|c|c|c|c|c|c|}
\hline Traits & $\mathrm{PH}$ & EH & MF & NP & $\mathrm{NE}$ & BP & LP & GY & ET & WS & $\mathrm{CS}$ & PP & $\mathrm{CZ}$ \\
\hline \multicolumn{14}{|c|}{ NAP5 - Location - Jataí GO } \\
\hline $\mathrm{NE}$ & 2.54 & 3.37 & -1.59 & 3.99 & 25.13 & 2.75 & 2.79 & 25.96 & -20.38 & -1.49 & -15.16 & -1.07 & 0.42 \\
\hline GY & 2.05 & 1.71 & -2.31 & 3.31 & 20.59 & 11.7 & -7.08 & 30.67 & -19.77 & -7.86 & -17.27 & 2.59 & -3.11 \\
\hline ET & 1.49 & 2.26 & -0.39 & -0.71 & 10.29 & 0.00 & -3.98 & 12.92 & -40.6 & -12.34 & -8.89 & -5.36 & -0.85 \\
\hline WS & -1.64 & -1.75 & -0.11 & 2.49 & 2.27 & 7.57 & 2.59 & 4.10 & -3.69 & -55.17 & -0.94 & -4.87 & -7.97 \\
\hline $\mathrm{CS}$ & 2.62 & 5.19 & -0.57 & 3.86 & 14.31 & 1.38 & 2.98 & 15.11 & -22.07 & -3.41 & -30.81 & -1.55 & -0.73 \\
\hline PP & 0.30 & 1.05 & 1.21 & -1.8 & -5.21 & -9.29 & 2.98 & -4.42 & -20.87 & -9.32 & 8.06 & -14.94 & -1.46 \\
\hline $\mathrm{CZ}$ & 0.26 & 1.11 & -0.02 & -0.5 & 3.88 & -6.54 & -0.12 & 5.56 & -10.68 & -13.46 & -13.02 & -3.29 & -19.78 \\
\hline Xo & 194.48 & 108.92 & 58.22 & 13.19 & 10.07 & 3.27 & 5.52 & 3.21 & 285.08 & 211.74 & 734.36 & 275.42 & 127.72 \\
\hline Xs & - & - & - & - & 14.24 & - & - & 4.50 & 138.09 & 75.93 & 426.31 & 201.22 & 79.58 \\
\hline \multicolumn{14}{|c|}{ NAP5 - Location - Uberlândia MG } \\
\hline $\mathrm{NE}$ & 2.95 & 2.49 & -0.97 & 2.49 & 14.50 & -8.23 & -2.05 & 15.65 & -12.92 & 2.68 & -10.11 & -0.30 & 2.43 \\
\hline GY & 1.64 & -1.43 & -1.28 & 0.85 & 5.97 & -2.27 & -9.21 & 29.97 & -8.67 & -1.81 & -16.56 & 0.00 & -2.14 \\
\hline ET & -0.34 & -3.98 & -1.31 & -0.26 & 3.68 & -6.06 & -4.43 & 10.84 & -36.3 & -11.83 & -14.26 & 3.19 & 2.36 \\
\hline WS & -0.84 & -1.55 & 0.90 & -1.70 & -2.40 & -7.15 & -2.73 & -5.26 & 0.85 & -44.89 & 10.91 & -1.85 & -0.98 \\
\hline CS & 3.08 & 2.21 & -1.07 & 1.45 & 3.38 & -6.06 & 9.55 & 12.13 & -7.34 & 15.17 & -28.72 & 3.34 & 0.57 \\
\hline PP & 0.45 & 1.49 & 1.14 & -0.62 & 0.56 & 0.97 & 4.43 & -3.09 & -4.73 & -5.66 & 3.29 & -9.83 & -1.67 \\
\hline $\mathrm{CZ}$ & -0.07 & -0.29 & 0.25 & 0.02 & 0.49 & -1.73 & 0.68 & -0.33 & 1.90 & 2.29 & 1.13 & -1.50 & -19.27 \\
\hline Xo & 191.08 & 106.60 & 59.17 & 17.34 & 13.94 & 1.67 & 2.49 & 1.97 & 464.39 & 372.41 & 817.60 & 237.97 & 90.32 \\
\hline Xs & 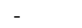 & - & - & - & 14.50 & - & - & 2.86 & 266.8 & 170.89 & 493.42 & 166.91 & 51.64 \\
\hline
\end{tabular}

PH: plant height (cm); EH: Ear height (cm); MF: male flowering (days); BP: number of broken plants; LP: number of lodged plants; GY: Grain yield ( $\mathrm{t} \mathrm{ha}^{-1}$ ); ET: Resistance to Exserohilum turcicum (AUDPC), WS (white spot): resistance to Pantoea ananatis (AUDPC), CS: resistance to corn stunt (AUDPC), PP: resistance to Puccinia polysora (AUDPC) and CZ: Cercospora zeae-maydis resistance (AUDPC).

With the selection of $20 \%$ of the superior progenies in both populations at both sites, the highest direct gains were obtained for the WS trait in the NAP5 population with negative estimates of 55.17 and $44.89 \%$ in Jataí, GO and Uberlândia, MG, respectively. For the NAP7 population the highest estimates were observed for the GY trait which presented the highest value in the Uberlândia test with $31.17 \%$ gain in grain yield. For estimates of indirect gains, the highest value of $25.96 \%$ for KW was observed when the selected trait was NE in the NAP5 population. The genetic gains obtained in the production of grains were higher than the values observed in studies with semiexotic populations of corn (Oliveira et al., 2015).

When direct selection for GY was performed, a gain was observed in reducing the severity of most diseases assessed in the NAP5 population and estimates varied according to the site evaluated, but the indirect selection for resistance may not be efficient due to the low path coefficients presented by the evaluated variables. 
Regarding the agronomic traits, direct selection for GY in the NAP5 population increased the number of broken plants in Jataí-GO by $11.7 \%$ and in the NAP7 population the number of plants in Uberlândia-MG increased by $8.55 \%$. In the cases of experiments in which the harvest is performed manually, all plants are harvested, including broken and root-lodged plants. However, when genotypes are grown in crops with mechanized harvest, broken and lodged plants can cause a high drop in grain yield. Therefore, it is necessary to make new evaluations for the BP and LP traits after the recombination of the selected families.

The variable NE presented cause and effect with GY, and it can be used for indirect selection, however the gain with direct selection provides greater gains than indirect selection. This result is due to the heritability estimate for GY (0.77) in the NAP5 population being higher than for NE (0.61).

Table 9. Estimates of Genetic Gain in percentage (GS\%) by the direct selection method for GY, ET, WS, CS, PP and CZ, average of the population (Xo) and average of the 20 selected families (Xs) in the exotic population of NAP-7 corn in Jataí, GO and Uberlândia, MG in 2015.

\begin{tabular}{|c|c|c|c|c|c|c|}
\hline Traits & GY & ET & WS & CS & PP & $\mathbf{C Z}$ \\
\hline & \multicolumn{6}{|c|}{ NAP7 - Jataí } \\
\hline GS $\%$ & 29.40 & -40.61 & -38.57 & -28.31 & -15.67 & -24.81 \\
\hline Xo & 4.45 & 365.81 & 248.74 & 709.23 & 295.95 & 142.63 \\
\hline Xs & 5.91 & 186.95 & 122.53 & 448.97 & 206.62 & 86.02 \\
\hline \multicolumn{7}{|c|}{ NAP7 - Uberlândia } \\
\hline GS\% & 31.17 & -18.48 & -22.11 & -18.33 & -17.78 & -19.78 \\
\hline Xo & 3.75 & 658.41 & 464.92 & 616.44 & 286.21 & 115.59 \\
\hline Xs & 5.03 & 469.93 & 301.38 & 411.02 & 182.70 & 66.27 \\
\hline
\end{tabular}

Table 10 shows the 15 families of full-sib selected in the NAP5 population and the 20 families selected for the NAP7 population in the two assessment sites for each variable. These families were selected for their productive potential and / or resistance to the evaluated plant pathogens by AUDPC. Bello et al. (2010) did the correlation and path coefficient analysis of yield and agronomic characters among open pollinated maize varieties and their F1 hybrids in a diallel cross, but didn't the correlations with diseases evolution by AUDPC (area under disease progress curve). Similar studies were done by Silva et al. (2016) but in this case the authors worked with correlation and path analysis of agronomic and morphological traits in maize.

In the NAP5 population of the various families selected, seventeen families presented superiority in three or more traits evaluated simultaneously, and the families $1,7,13,20,24$ and 57 stood out because they were superior in the two evaluated sites. For the NAP7 population, the number of families with these characteristics was 20 and those with superiority in the two sites were in families $18,22,45,77$ and 95. Juliatti et al 2004, 2009 and 2013 showed the possibilities of selection for plant pathogen resistance in an endogamic maize population for the main diseases (cercospora leaf spot, common rust and white spot). 
Table 10. Full-sib progenies selected by direct selection method of GY, ET, WS, CS, PP and CZ variables in two corn populations.

\begin{tabular}{|c|c|}
\hline Traits & NAP5 - Jataí- Progenies \\
\hline NE & $5,6,8,13,14,16,23,26,38,39,49,50,57,59,73$ \\
\hline GY & $1,5,6,7,8,16,23,26,27,39,49,50,57,58,73$ \\
\hline ET & $1,3,5,7,16,18,20,23,24,25,38,49,53,57,59$ \\
\hline WS & $1,5,9,12,13,20,22,24,42,50,55,65,73,74,75$ \\
\hline $\mathrm{CS}$ & $1,3,5,7,13,19,23,28,30,38,41,45,57,59,73$ \\
\hline PP & $4,14,18,20,24,34,41,42,46,49,52,55,59,71,72$ \\
\hline \multirow[t]{2}{*}{$\mathrm{CZ}$} & $1,2,4,5,13,21,24,31,34,37,39,41,44,49,68$ \\
\hline & NAP5 - Uberlândia- Progenies \\
\hline $\mathrm{NE}$ & $1,6,13,14,23,24,38,39,41,45,49,54,57,63,72$ \\
\hline GY & $11,23,24,27,30,34,43,45,46,54,57,69,72,73,74$ \\
\hline ET & $1,3,5,11,20,23,24,27,36,39,43,49,57,68,69$ \\
\hline WS & $1,2,4,9,13,20,22,24,29,42,50,54,62,69,75$ \\
\hline CS & $16,18,19,27,33,36,38,43,45,48,50,57,65,70,73$ \\
\hline PP & $1,6,8,12,13,14,18,20,22,26,37,44,51,54,70$ \\
\hline \multirow[t]{2}{*}{$\mathrm{CZ}$} & $3,8,9,13,14,16,21,24,29,43,44,55,66,70,74$ \\
\hline & NAP7 - Jataí- Progenies \\
\hline GY & $3,10,15,19,23,25,29,30,37,45,47,51,52,58,60,67,69,77,92,95$ \\
\hline ET & $3,7,10,18,26,27,28,29,44,55,58,61,74,78,82,87,88,93,95,96$ \\
\hline WS & $3,5,9,17,21,22,28,30,38,44,45,58,63,69,77,82,83,88,95,98$ \\
\hline CS & $1,2,4,7,19,22,23,34,38,44,45,51,55,57,75,77,80,82,93,95$ \\
\hline PP & $1,9,12,17,18,21,22,26,28,37,40,43,46,58,62,64,67,82,83,99$ \\
\hline \multirow[t]{2}{*}{$\mathrm{CZ}$} & $3,5,12,18,19,21,22,27,43,46,47,52,59,61,62,74,76,84,86,93$ \\
\hline & NAP7 - Uberlândia- Progenies \\
\hline GY & $2,4,18,20,27,28,29,31,33,35,38,56,60,62,67,69,70,77,79,95$ \\
\hline ET & $3,7,11,17,18,22,27,29,38,44,58,63,66,69,70,74,85,87,88,95$ \\
\hline WS & $5,15,22,28,29,38,44,45,51,61,63,67,69,73,75,77,82,83,84,88$ \\
\hline CS & $7,11,19,20,23,26,29,36,39,45,55,57,59,60,65,66,77,82,85,95$ \\
\hline PP & $3,4,7,21,22,25,27,30,37,40,43,49,57,62,64,65,71,73,74,92$ \\
\hline $\mathrm{CZ}$ & $1,4,7,8,12,14,18,22,41,45,46,47,52,59,68,72,73,84,87,93$ \\
\hline
\end{tabular}

\section{CONCLUSIONS}

In all the trials, the influence of the environment was low due to the predominance of genotypic correlations in relation to the phenotypic correlations. The phenotypic, genotypic correlations and the path analysis in the NAP5 population indicated the variable number of ears as having the greatest favorable effect on grain yield in corn. For the NAP5 population the indirect selection for yield through the selection of the number of ears can be useful and the genetic gain satisfactory. The NAP7 population had low phenotypic and genotypic correlations between the evaluated variables and grain yield (GY). With the use of the direct selection method it is possible to obtain gains of up to $31 \%$ in grain yield of corn in the evaluated populations. In the NAP5 population of the various families selected, seventeen families presented superiority in three or more traits evaluated simultaneously, and the families 1, 7, 13, 20,24 and 57 stood out because they were superior in the two evaluated sites. For the NAP7 population, the number of families with these characteristics was 20 and those with superiority in the two sites were in families 18, 22, 45, 77 and 95. The variable NE had a cause and effect relationship with GY, and it can be used for indirect selection; however, the gain with direct selection provided greater gains than indirect selection. This result is due to the heritability estimate for GY (0.77) in the NAP5 population being higher than for NE (0.61). Plant pathogen resistance was obtained in both populations against Cercospora leaf spot, common rust and white spot and resistance to Exserohilum turcicum and Physopella zeae. 


\title{
ACKNOWLEDGMENTS
}

The authors thank CNPq, CAPES, FAPEG, FUNDAP - UFU and FAPEMIG for financial support and Professor José Branco Miranda Filho - UF Jataí GO "in memoriam" for all the scientific advice and friendship during the research projects.

\section{CONFLICTS OF INTEREST}

\author{
The authors declare no conflict of interest.
}

\section{REFERENCES}

Agroceres (1996). Guia agroceres de sanidade. Sementes Agroceres, São Paulo. 72 p.

Bello OB, et al. (2010). Correlation and path coefficient analysis of yield and agronomic characters among open pollinated maize varieties and their F1 hybrids in a diallel cross. Afr. J. Biotechnol. 9: 2633-2639.

Brito AH, Von Pinho RG, Pozza EA, Pereira JLAR, et al. (2007). Efeito da cercosporiose no rendimento de híbridos comerciais de milho. Fitopatol. Bras. 32(6): 472-479. https://doi.org/10.1590/S0100-41582007000600004

Cabral PDS, do Amaral Júnior AT, de Jesus Freitas IL, Ribeiro RM, et al. (2016). Relação causa e efeito de caracteres quantitativos sobre a capacidade de expansão do grão em milho-pipoca. Rev. Ciênc. Agron. 47(1): 108-117.

Companhia Nacional de Abastecimento (CONAB) (2020). Acompanhamento de safra brasileira: grãos, sétimo levantamento, abril 2018. Available at: <http://www.conab.gov.br>. Accessed April 16, 2020.

Cota LV, Costa RV and Silva DD (2015). Manejo de doenças. In: Borém, A.; Galvão, J. C. C.; Pimentel, M. A. Milho: do plantio à colheita. 1.ed. UFV, Viçosa. pp. 294-322.

Cruz JC, Silva GH, Pereira Filho IA, Gontijo Neto MM, et al. (2011) Sistema de produção de milho safrinha de alta produtividade: Safras 2008 e 2009. Embrapa, Sete Lagoas, 10.

Cruz CD, Regazzi AJ and Carneiro PCS (2012). Modelos biométricos aplicados ao melhoramento genético. 4. ed. Viçosa: Imprensa Universitária, v. 1, 514 pp.

Cruz CD (2016). Genes Software-extended and integrated with the R, Matlab and Selegen. Acta Sci. Agron. 38(4): 547552. https://doi.org/10.4025/actasciagron.v38i3.32629

Dudienas C, et al (2013) Severidade de ferrugem pollissora em cultivares de milho e seu efeito na produtividade. Summa Phytopathol. 39: 16-23. https://doi.org/10.1590/S0100-54052013000100003

Entringer GC, Santos PHAD, Vettorazzi JCF, Cunha KS, et al. (2014). Correlação e análise de trilha para componentes de produção de milho superdoce. Rev. Ceres. 61: 356-361.

Fancelli AL and Dourado Neto D (2000). Produção de Milho. Guaíba: Agropecuária. 360.

Ferreira A, Cruz CD, Vasconcelos ES, Nascimento M, et al. (2008). Utilização de bootstrap não-paramétrico para avaliação de correlações fenotípicas, genotípicas e ambientais. Acta Sci. Agron. 30: 657-663.

Juliatti FC, Pedrosa MG, Juliatti BCM, Beloti IF, et al. (2013). Identificação de QTLs associados à resistência parcial à mancha branca do milho. Biosci J Uberlândia. 29 (5): 1163 -1178.

Juliatti FC, Appelt CCNS, Brito CH, Gomes LS, et al. (2004). Controle da feosféria, ferrugem comum e cercosporiose pelo uso da resistência genética, fungicidas e épocas de aplicação na cultura do milho. Biosci. J. Uberlândia. 20(3): 45-54.

Juliatti FC, Pedrosa MG, Silva HD and Silva JVC (2009). Genetic mapping for resistance to gray leaf spot in maize . Euphytica. 169: 227-238. https:// doi 10.1007/s10681-009-9943-2.

Lazaroto A, Santos I, Konflanz VA, Malagi G, et al. (2012). Escala diagramática para avaliação de severidade da helmintosporiose comum em milho. Cienc. Rural. 42: 2131-2137. https://doi.org/10.1590/S010384782012005000112.

Li CC (1975) Path analysis: a primer. Boxwood: Pacific Grove. 346.

Malagi G, Santos I, Camochena RC and Moccellin R (2011). Elaboração e validação da escala diagramática para avaliação da mancha branca do milho. Rev. Ciênc. Agron. 42: 797-804. https://doi.org/10.1590/S180666902011000300028.

Mendes UC, Miranda Filho JB, Oliveira AS and Reis EF (2015). Heterosis and combining ability in crosses between two groups of open-pollinated corn populations. Crop Breed Appl. Biotechnol. 15: 235-243. https://doi.org/10.1590/1984-70332015v15n4a40.

Montgomery DC and Peck EA (1982). Introduction to linear regression analysis. J. Wiley, New York. 504 p.

Oliveira E, Fernandes FT, Casela CR, Pinto NFJA, et al. (2004). Diagnose e Controle de Doenças na Cultura do Milho. In: GALVÃO, J. C. C.; MIRANDA, G. V. Tecnologia de Produção do milho: economia, cultivares, biotecnologia, safrinha, adubação, quimigação, doenças, plantas daninhas e pragas. UFV, Viçosa. chapter 7. 
Oliveira AS, Reis EF, Miranda Filho JB, Mendes UC, et al. (2015). Genetic variability and yield potential of three semiexotic corn (Zea mays L.). Biosci. J. 31: 1609-1617. https://doi.org/10.14393/BJ-v31n6a2015-28783.

Shaner G and Finey RF (1977). The effects of nitrogen fertilization on the expression of slow-mildewing resistance in Knox wheat. Phytopathology. 67: 1051-1056. https://doi.org/10.1094/Phyto-67-1051632188.

Silva, TN, Moro, GV, Moro, FV, Santos, DMMD, et al. (2016). Correlation and path analysis of agronomic and morphological traits in maize. Rev. Ciênc. Agron. 47(2): 351-357.

Singh ER and Chaudhary BD (1979). Biometrical methods in quantitative genetic analysis. Kalyani Publishers, New Delhi. 304 p.

Toebe M and Cargnelutti Filho A (2013). Não normalidade multivariada e multicolinearidade na análise de trilha em milho. Pesq. agropec. bras. 48: 466-477. https://doi.org/10.1590/S0100-204X2013000500002.

Vencovski R and Barriga P (1992). Genética biométrica no fitomelhoramento. Sociedade Brasileira de Genética, Ribeirão Preto. 496 pp.

Wright S (1921). Correlation and causation. J. Agric. Res. 20: 557-585. 